\title{
artículos
}

\section{La síntesis crítica del conocimiento bibliotecológico: su valor para la Investigación en Bibliotecología.}

\author{
Ramiro Lafuente \\ Investigador del CUIB
}

\section{RESUMEN}

Se pro pone la ela bo ra ción de sín te sis críti cas — au to ri za das por su ni vel aca dé mi co- con ob je to de re va lo rar y re plan tear los pro ce dimien tos de in ves ti ga ción bi blio te co ló gi ca, sobre todo aquellos orien ta dos a la in ves ti ga ción mis ma, que no res pon den a fi nes uti li ta rios sino a bus car la ex pan sión del co no ci mien to, y sus me tas no es tán en ca mi na das a lo gros de cor to pla zo o a in ten tos tec no ló gi cos por mejo rar un pro ce so o servi cio. La in ten ción es re ca pi tu lar in for ma ción re la ti va a los co m po nen tes bá si cos de la bi blio te ca y sus ne xos con la so cio di ná mi ca cul tu ral, para ha cer cla ras y for ma les las li gas en tre di ver sos hechos que afec tan la vida bi blio te ca ria para ob te ner una explicacióncoherente de los mis mos y faci li tar el or de na mien to de la in ves ti ga ción bi blio te co ló gi ca y la co m pren sión de los fe nó menos bibliotecarios.

\section{SUMMARY}

The ela bo ra tion of criti cal synthe ses is pro po sed — autho ri zed at the aca de mic le vel—w ith the obje ti ve of re va lua ting and res ta ting the procedues of li brary re search, abo ve all tho se orien ted toward re search itself, that do not res pond to uti li ta rian goals but the search for the ex pan sion of knowledge. Its aims are not di rec ted at short-term achie ve ments or at te chnological at tempts for im pro ving a process or service. The intention is to summarize information relative to the basic components of the library and its links with sociocultural dyna mics. This should make clear and re lia ble the bonds bet ween di vers fac tors that effect li brary life in or der to ob tain a cohe rent explanation of these links and thus facilitate the ordering of library research and the comprehension of library phenomena.

El origen de las técnicas para organizar manuscritos y testimoniosdocumentales es tan antigua como la invención de la escritura. $\mathrm{Su}$ organización como disciplina tiene viejas raíces y durante muchotiem pofueconsideradaexclusivamen tecomoun arte utilitario para realizar catálogos y organizar documentos e impresos.

Sólo hasta tiempos relativamente recientes la bibliotecología empezó a generar un cuerpo de conocimientos teóricos, útiles para comprender la naturaleza de los fenómenos de comunicación y transmisión del conocimiento que se da en torno a las bibliotecas. Empero, generalmente el bibliotecario, durante el ejercicio profesional, pone escaso interés en aspectos de comuni ca ción del co no ci mien to pro pios de la bi blio te ca y opta por envol ver se en el ro paje que le pro por cio na la or ga ni za ción téc ni ca bibliotecaria.

Elén fa sis en proble mas de or ga ni za ción in terna de la bi blio te ca, se ha re fle ja do en lain ves ti ga ción bi blio te co ló gi ca aboca da fundamentalmente a buscar aplicaciones técnicas especializadas con el propósito de solucionar cuestiones prácticas del trabajo biblio te ca rio, en de tri men to de la com pren sión del pa pel de la biblioteca como parte de la red global de comunicación humana.
La ten den cia a su pe di tar lain ves ti ga ción bi blio te co ló gi ca y arreglo de bibliotecas a la naturaleza téc ni ca de los he chos bi blio tecarios, se expresa desde fines del siglo XIX, por medio del uso fre cuen te de con cep tos como: "pun to de vis ta cien tífi co" y ad ministración "científica" de las bibliotecas, con una particular insistencia en la búsqueda de la "eficiencia" (véase Casey).

Este modo de con ce bir la bi blio te co lo gía for ma par te dellen guajey con cepciones teóricas co munes delbiblio te cario profe sional y ha ser vi do para sus ten tar fir me men te la idea de que el ob je ti vo pri mor dial de la bi blio te co lo gía es crear y ma ne jar pro ce di mientos técnicos para organizar bibliotecas. Creencia además compartida por otros círculos sociales.

Considerar a la bibliotecología exclusivamente como un saber técnico, reduce considerablemente los horizontes de investigación bi blio te co ló gi ca. Para am pliar los y abrir nue vas po si bi lidades deinves tigación, resul ta particu larmentevalioso, el or de nar y sin te ti zar la in for ma ción exis ten te, parafun da men tar unconocimiento bibliotecológico, que vincule aspectos técnicos específicos corres pon dientes a laes truc turay for mas de organización de servi cios bi blio te ca rios con aque llos fe nó me nos so cia les que influyen y/o determinan el desarrollo de bibliotecas. 
Destacar los aspectos sociales de los fenómenos bibliotecarios tendría como finalidad buscar una adecuada correspondencia en tre el obli ga do sa ber téc ni co del bi blio te ca rio y los pro ble mas inherentes a la circulación documental, para precisar desde una perspectiva de organización bibliotecaria, las conexiones entre la es truc tu ra crea da por una o va rias bi blio te cas y los com po nentes básicos que intervienen en la comunicación de conocimientos, a saber:

las costumbres adoptadas para la circulación de textos o de cualquier otro medio de difusión de conocimientos;

elva lor con ce di do por una co mu ni dad o un cír cu lo so cial a determinados materiales documentales;

las for mas en que se je rar qui zan los di ver sos me dios de di fusión de información en una comunidad determinada;

los fines institucionales y/o sociales de las bibliotecas.

Así como aque llos as pec tos cua li ta ti vos que se pre sen tan en torno a la bi blio te ca como ins tru men to de pre ser va ción de todo tipo de materiales y documentos (véase Nitecki).

Al abordar los distintos fenómenos que se dan en torno a la biblioteca como instrumento de preservación y comunicacióndocumental, no se debe perder de vista el papel que juega el biblio te cario en la for ma ción de co lec cio nes de ma te ria les do cur men ta les del pre sen te y del pa sa do. Ma te ria les que ad quie ren un va lor úni co al ser acu mu la dos en un solo es pa cio fí si co, para faci li tar su dis po ni bi li dad irres tric ta a di fe ren tes sec to res de la población en cualquier momento. Un tema central puede llegar a ser el pa pel del bi blio te ca rio como guía del con te ni do de la in formación registrada por la biblioteca (Mansfield, p. 66).

El sintetizar el conocimiento bibliotecológico, es una tarea que obliga a reunir, evaluar y encadenar los fenómenos propios del quehacerbiblio te cario paraes tablecer lo que real men te sabe mos acerca de las biblio te cas, sus proce di mien tos téc ni cos y fi nalidades de sus ser vi cios. Así como el si tio que ocu pan den tro de una comunidad y el valor que representa la biblioteca en calidad de salvaguarda de materiales constitutivos de la memoria documental de una sociedad.

Elpre pararsín te sis so bre dis tin tos as pec tos del conocimien to bi bliotecológico facilitaría la formación de marcos de referencia úti les para de ter mi nar las re la cio nes en tre las téc ni cas de or ga nización bibliotecaria y la sociodinámica de la circulación de información en una determinada comunidad. Además estas síntesis permitirían ob te ner una vi sión glo bal de la bi blio te co logía y sus características esenciales como un todo que facilitesu apropiación y difusión.

La ela bo ra ción de sín te sis críti cas -a ut or iz adas por su ni vel académico- requiere revalorar y replantear los procedimientos de investigación bibliotecológica, sobretodo aquellos orien ta dos a la in ves ti ga ción mis ma, que no res pon den a fi nes uti li ta rios sino a bus car la ex pan sión del co no ci mien to, y sus me tas no es tán encaminadas a logros de cor to pla zo o a in ten tos tec no ló gi cos por mejorar un proceso o servicio.
La función primaria de estas síntesis sería obtener una explicación clarificadora, consistente en sis te ma ti zar he chos ya co nocidos, y no en proporcionar razones para creer en hechos que conocemos por otras fuentes (la experiencia o la autoridad).

La re ca pi tu la ción de in for ma ción re la ti va a los com ponen tes bá si cos de la bi blio te ca y sus ne xos con la so cio di ná mi ca cul tu ral, no generaría nuevos datos sobre el mundo de las bibliotecas, su in ten ción al sis te ma ti zar la in for ma ción dis po ni ble, se ría ela borar una explicación acer ca de fe nó me nos bi blio te ca rios, para insertarlos en el conjunto de creencias existentes, estructuradas y justificadas, sobre la organización bibliotecaria.

Explicar cualquier fenómeno relacionado con el quehacer biblio te cario ten dría el pro pó si to de en con trar vin cu la cio nes en tre va rios he chos y su ce sos en tre sí, con ob je to de ha cer los cohe rentes. La coheren cia en tre cre en cias es el re sul ta do epis te mo ló gi co de la explicación (Ponce, p. 51).

El valor metodológico de una síntesis crítica radicaría en hacer cla ras y for ma les las li gas en tre di ver sos fe nó me nos que afec tan la vida bibliotecaríapara obtener una explicacióncoherente de los mismos y facilitar con ello el ordenamiento de la investigación bi blio te co ló gi ca y la com pren sión de los fe nó me nos bibliotecarios.

\section{La explicación te leo ló gi ca como mé to do para la ela bo ra ción de síntesis críticas del conocimiento bibliotecológico}

La mo ti va ción para efec tuar ex pli ca cio nes acerca delfe nó me no bibliotecario no ra di ca en cues tio nes prác ti cas, sino en bus car la in te li gibilidad de un he cho, tie ne un carác terclarifica dor y for ma par te esen cial de su ra zón de ser la de ter mi na ción de com pren der un fenómeno. Como indica Hempel, nace del "profundo y persis ten te de seo de com pren der se a sí mis mo y a su mun do" (Hempel, p. 333).

Un su je to pue de com pren der un fe nó me no o un su ce so en múl tiples for mas, pero en úl ti mo tér mi no, la ex pli ca ción que ela bo ra rá del su ce so o fe nó me no en cues tión es ta rá en ra zón de aque llo que quiere encontrar o justificar. El tipo de explicación depende de cómo un su je to de see co nec tar el fe nó me no des cri to con otros fenómenos, de sus intereses cognoscitivos y de las circunstancias que lo motivan a buscar una explicación concreta.

Puede existir previamente un interés por conocer las causas de un fe nó me no, e in cli nar se por una ex plica ción causal. Tam bién es factible tratar de comprender un fenómeno como una totalidad, como la suma o com bi na ción de cier tas par tes y ge ne rar una micro explicación.

Otra op ción para ex pli car un fe nó me no es tra tar de com pren der sus "consecuencias" y obtener una explicación teleológica (Pon ce, p. 55). En este caso no son re le van tes para la ex pli ca ción los an te ce den tes (cau sas o par tes) de un fe nó me no, sino losefectos que éste produce. 
La explicación causal persigue establecer principios generales para predecir el comportamiento futuro de los hechos, parte de las pre mi sas y de du ce la con clu sión; en cam bio, en el pro ce so de explicaciónteleológica se co no cen los he chos y de allí se pos tulan las premisas que lo justifican.

La ex plica ción te leo ló gi ca de un fe nó me no, a par tir de sus con secuencias o resultados, permite establecer sus diversos nexos y saber, por ejemplo, de dónde proviene, cuál es su composición, qué con se cuen cias tie ne su ope ra ción, etc. Exis ten he chos y/o fenómenos bibliotecarios cuya explicación únicamente puede conseguirse a través del análisis de sus resultados o consecuencias, es de cir, por me dio de una explicación teleológica que los haga in te li gi bles. Es por ejem plo el caso de los ser vi cios bi blio tecarios, la catalogación o el uso de normas.

Un hecho que se desea explicar se vuelve inteligible cuando se fija una co ne xión (o co ne xio nes) con otra cosa que lo de ter mi na de al gu na ma ne ra. "En vir tud de esa(s) co ne xión(es), el he choen cues tión se ve, o bien, como cau sa o efec to de esa otra cosa que lo de ter mi na, o bien como una to ta li dad com pues ta de"n" partes , o como un sis te ma que po see de ter mi na da es truc tu ra, etc." (Ponce p. 52).

Alins ti tuir una co rre la ción en tre el fe nó me no des cri to y aque llo que lo de ter mi na, se es table ce tam bién un prin ci pio ge ne ral, que valida las relaciones explicativas.

El va lor ex pli ca ti vo de un ar gu men to dado se mide por su po der para ha cer más cohe ren tes nues tras cre en cias acer ca de un fe nómeno determinado.

Para realizar una síntesis, sabemos las consecuencias o resultados de algunos fenómenos bibliotecarios, pero no cómo evolucio na ron ni el por qué de esa evo lu ción. Con la ex pli ca ción no se tra ta ría de ob te ner una res pues ta uní vo ca a esa evolu ción, sino un planteamiento ordenado de acontecimientos y problemas que permita entender el proceso de transformación de los hechos o fenómenos bibliotecarios.

Un planteamiento ordenado de acontecimientos y problemas acercade un fe nómenobibliotecario, produciría una ex plicación coherente del mismo -si se quiere una bien fundada teoría- que establezca relaciones de diacronía y sincronía con otros fenómenos, y desde la cual podamos deducir las características del fenómeno que estudiamos.

Las relaciones de diacronía de un hecho o fenómeno permiten trans for mar la in for ma ción dis per sa en una se cuen cia de he chos o su ce sos para con fi gu rar un pa trón in te li gi ble. Su obje ti vo es seguir el desenvolvimiento de un hecho para resaltar su proceso evolutivo.

Como los he chos su ce den en un lap so de tiem po y sus efec tos tienen dis tin taduración, las re la ciones de sincronía facilitanenlazar un conjunto de hechos dentro de un marco de referencia cronológico. Sufinali dades crear paráme tros cro no ló gi cos para interconectar entre sí, un conjunto de hechos que funcionan al mismo tiempo.

Elordenamien to de los fe nóme nos biblio teca rios pue de ayu dara construir marcos de referencia para jerarquizar el conocimiento bibliotecológico (que permita manejar la diacronía y sincronía de los fenómenos bibliotecarios). La cons truc ción de mar cos de refe ren cia cons ti tu ye una he rra mien ta ló gi ca para en cau sar la investigación, al proveer un sistema de ordenación de creencias comunes respecto de los fenómenos propios de la organización bibliotecaria.

Los marcos de re fe ren cia ten drían como pro pó si to pro por cionar ele men tos in dis pen sables para que cual quier su je to pue dacap tar el sig ni fi ca do de las des crip cio nes de una ex pli ca ción. Al ca racterizar un hecho o fenómeno bibliotecario se utilizan términos téc ni cos para des cribirlo: "catálogos", "conteni dos semánticos", "descriptores", "control biblio-gráfico", "evaluación de servicios", etc. Cada término tiene una determinada connotación y únicamente es posible comprenderlo en relación con un marco de referencia donde se proporcione su sentido, vinculaciones con otros fe nó me nos, y su con te ni do con cep tual paraiden tifi car los problemas involucrados en el mismo.

"Di cho de otro modo, la des crip ción mis ma de los he chos y su cesos es po si ble sólo des de la pers pec ti va de quien pue de iden ti ficar los tér mi nos que fi gu ran en ella y en las re la cio nes que se dan entre tales hechos y los sucesos" (Ponce, p.27). No es posible ofrecer explicaciones acerca de hechos determinados a quienes no po sean un mar co con cep tual ade cua do para co lo car den tro de él tal hecho.

Una explicación no puede ser buena para todos, porque aunque dos su je tos ten gan los mis mos mar cos con cep tuales, el con jun to je rarquiza do de sus cre en cias pue de ser dia me tral men te opues to, y puede suceder que aunque la explicación no cambie para ningu no de los dos, sí pue decam biar la im por tan cia de la ex pli cación ofrecida de acuerdo con la perspectiva desde la cual se le considere (Ponce, p. 30).

En el caso de una ex pli ca ción ge ne ral don de se in vo lu cran va rios fe nó me nos, un su je to pue de fi jar se en un he cho par ti cu lar per teneciente a esa explicación general, y reunir fenómenos que pertenecen a distintas áreas de su interés. Estos fenómenos que se estructurarían coherentemente dentro de ámbitos predefinidos, en tran en con flic to tan pron to como se les ubi ca en uno dis tin to.

Aunque todos los fenómenos bibliotecarios interaccionan entre sírecíprocamente, no de be mos re la ti vi zar en ex ce so su des cripción. Alex plicar los es im pres cin dible con si de rar los como sistemas aislados coordinados entre sí en un conjunto ordenado de hechos que contribuyen a producir determinados efectos, para evitar perdernos en un infinito de interacciones universales.

La bi blio te ca es unorganis moencrecimiento, constantemente ali men ta do por la di ná mi ca cul tu ral de pro duc ción y trans for mación de materiales documentales de todo tipo (Vickery, p.8). 
Cuando una biblioteca no participa de esa dinámica cultural, cambia su naturaleza y connotación social.

Esta característica de la biblioteca, la de ser un organismo en constan te crecimien to, hace que los fe nóme nos bibliotecarios, al consi de rar los como obje to de es tu dio, se pre sen ten como he chos continuos, estrechamente unidos entre sí.

El to mar como base para de li mi tar y re pre sen tar un fe nó me no biblio te cario, las con se cuen cias que éste produce, per mi te ais lar lo y considerarlo como un evento discreto, compuesto de un conjun to de par tes di ver sas su je tas a un pro ce so evo lu ti vo en un ámbito predefinido de referencias cronológicas.

El sin te ti zar fe nó me nos bi blio te ca rios a par tir de sus con se cuencias o efectos, fa ci li ta co rre la cio nar di ver sas cues tiones vinculadas a un fenómeno en particular, englobándolo en una sola explicación.

Como la sín te sis se rea li za ría unien do en un cuer po cohe ren te la miscelánea informativa producto de diversos estudios técnicos so bre or ga ni za ción y fines bi blio te ca rios, ten dría por obje to buscar que la pluralidad de en fo ques y mo de los pre sen tes en los estu dios téc ni cos ana li za dos, pue dan in te grar se en unideal teó ri co articulado.

Este ideal teórico articulado proporcionaría los elementos suficientes para delimitar los fines y propósitos de la estructura bibliotecaria en un contexto social determinado, con lo cual la pluralidad de formulaciones de problemas y enfoques técnicos pue de ubi car se den tro de mar cos de re fe ren cia cla ra men te ex plí ci tos; re du cien do las dis pu tas so bre los fun da men tos teó ri cos de la bi blio te co lo gía y sus re la cio nes con las prác ti cas de in ves ti gación ac tua les, al crear cri te rios de fi ni dos para or de nar la in ves tigación sobre fenómenos bibliotecarios.

\section{La biblioteca como objeto de estudio}

Constituye una unidad -un todo-, forma una estructura cuyos componentes se determinan entre sí, tanto en sus funciones como en el or den y dis po si ción de sus pro pie da des. Esta in te racción recíproca entre los componentes de la biblioteca se da en vir tud de prin ci pios ge ne ra les de na tu ra le za téc ni ca, o bien obedece a requerimientos de tipo social.

Algu nos de los prin ci pios ge ne ra les o le yes del fun cio na mien to de las es truc tu ras bi blio te ca rias, sólo ad quie ren sen ti do en ra zón de sus fines o consecuencias sociales dentro del ámbito de concepciones téc nicas o ideo ló gi cas pro duc to de la di ná mi ca cul tural de una organización social determinada.

"La bi blio te ca es qui zá el con cep to más es tu dia do, y con pe queñas varia cio nes, loen con tra mos de fi ni do de for ma muy téc nica." (Ruiz, p. 5).

La conceptualización técnica de la biblioteca conduce en gran medida a apreciaciones cerradas que tratan de "corresponderse exac ta men te sin nin gu na fi su ra a la fun ción clá si ca de la bi blio te- ca” (Ruiz,p. 5). Man te ner una idea ex clu si va men te téc ni ca de la bi blio te ca, con tribu ye en gran me di da a frag men tareles tu dio de la misma, al crear universos técnicos desvinculados de la función glo bal de la bi blio te ca y de las ca rac te rís ti cas que le im prime la dinámica del desarrollo cultural.

Una de las fun cio nes de la sín te sis de in for ma ción so bre fe nó menos bibliotecarios, sería integrar un conocimiento sobre la biblioteca alejado de concepciones restrictivas derivadas del es tu dio in de pen dien te de cada uno de sus as pec tos. El sen ti do de las sín te sis es ta ría en de ter mi nar los con cep tos de unión en tre las necesida des propias de la or ganiza ción téc nicabiblio tecariay las finalida des sociales de la produc ción y circu la ción de ma te riales documentales.

Se pa rar y des vin cu lar toda la gama de fe nó me nos que afec tan la vida de la bi blio te ca, ha te ni do la in ten ción de man te ner parce las de estudio más con fines corporativos que científicos, para ello se buscan constantemente razones inexistentes para evitar consolidar en una sola materia de estudio todos los fenómenos que se dan en torno a la biblioteca (véase Ruiz).

Cons truirsín te sis de in for ma ción re fe ren te a cues tiones vin cu la das al quehacer bibliotecario, requiere categorías que presten considerable atención a los juicios cualitativos y de detalle, indispensables para hacer explícitas las concepciones teóricas o ideológicas que intervienen en la descripción de fenómenos bibliotecarios (véase Olivé).

Ex pli car al gún he cho o fe nó me no bi blio te ca rio, sin apo yar se en juicios cualitativos sobre la función de la biblioteca como un todo, conduce a la fragmentación de los fenómenos bibliotecarios, reduciendo la explicación a funciones técnicas aisladas de cualquier contexto o connotación social.

Sin te ti zarin for ma ción de ri va da de es tu dios so bre cues tiones bi bliotecarias, requiere tener presente que los modelos teóricos que se utilizan, así como sus esquemas de clasificación asociados (como por ejemplo, la tipificación técnica de bibliotecas en especializadas, públicas, etc.), permanecen firmemente ligados a hechoshumanosimportantes, re la cio na dos con las for mas de organización y disponibilidad social del conocimiento por medio de ins ti tu cio nes pú bli cas como bi blio te cas, cen tros de in formación, archivos, redes de comunicación, etc.; sobre las cuales no existe un ideal claramente articulado para ordenarlos, que pueda ser utilizado como marco de referencia teórico para integrar una visión global de la biblioteca.

Los ideales de organización bibliotecaria generalmente se limitan a mencionar las bondades de los procedimientos técnicos para alcanzar metas específicas, por ejemplo, se destacan las cualidades del catálogo como instrumento de comunicación biblioteca-usuario, sin escudriñar en las concepciones y contenidos ideológicos implícitos en la organización y clasificación catalográfica, que determinan la eficacia del catálogo.

Ana li zar he chos y fe nó me nos bi blio te ca rios para ob te ner ex plica ciones acer ca de cues tio nes inhe ren tes a la bi blio te ca, ubi cán- 
dola en contextos relativos a su naturaleza de instrumento de circulacióny memoria documental, presentamúltiples dificultades. Una de las mayores estriba en delimitar rigurosamente los objetos de estudio para evitar superposiciones entre ellos, derivadas de diferentes enfoques, al involucrarseen el análisis concepciones técnicas y fenómenos sociales, lo cual supone la existencia de un mar co de re fe ren cia que ca rac te ri ce los problemas y vínculos de determinado fenómeno bibliotecario.

Las for mas que pue de adop tar la téc ni ca de or ga ni za ción bi bliotecaria se encuentran vinculadas a diversas concepciones sobre las finalidades sociales de la biblioteca; de ahí la necesidad de que la ela bo ra ción de sín te sis críti cas del co no ci mien to bi blio tecológico estén fundamentadas en un conjunto de axiomas que nos permita visualizar las vinculaciones entreorganización técnica y las finalidades sociales de la estructura bibliotecaria.

Estos axiomas estarían constituidos por los siguientes principios:

a) Labiblio te ca es un or ga nis mo en cre cimien to, cons tan te men te alimentado por la dinámica de producción y transformación de materiales documentales de todo tipo.

b) La bi blio te ca es un or ga nis mo de na tu ra le za so cial, que pue de adop tar di ver sos mo dos o ma ne ras de ser, de acuer do a sus me tas y al valor que se le atribuya a los materiales documentales que maneja.

c) Los servicios bibliotecarios son una consecuencia de las formas que adopta la estructura técnica de la biblioteca.

Unabiblio te ca cons ti tu ye una es truc tu raúnica, su je ta a una di ná mica social que puede hacer variar sus objetivos inmediatos, pero su naturaleza esencial no se transforma sustancialmente. Va rían sus ser vi cios y fi na li da des so cia les en ra zón de su his to rici dad, a tra vés del tiem po se le han asig na do fi nes y me tas con siderados como valiosos por un sistema de organización social determinado.

Por sus objetivos a cumplir se distinguen diversos tipos de bibliotecas:universitarias, públicas, escolares, etc.Latipificación biblio te caria tie ne un gran con te ni do de his to ri ci dad, laidea de la biblio te ca como servi cio pú bli co se ge ne ró du ran te el sur gi miento del liberalismo y siguió su propia evolución, así como la biblio te ca téc nica es pe cia li za da crea da a par tir del de sa rro llo de la cien cia mo derna; esta his to ri ci dad de ter mi na los mo dos o ma neras de ser de la biblioteca, adoptados a lo largo de su desarrollo histórico.

Algunos proble mas bibliotecariosúnicamentees factibleentender los y ex pli car los en ra zón de sus con se cuen cias. Por ejem plo, los ser vi cios que pres ta una bi blio te ca, son una con se cuen cia de las formas que adopta la organización técnica de la misma, por tan to el aná li sis y com pren sión de esos ser vi cios, nos re mi te a la estructura bibliotecaria que los produce.La explicación que podríamos ofrecer sobre los servicios bibliotecarios siempre sería relativa y estaría en correlación con la estructura organizativa que guarda la biblioteca que los realiza.

Los fi nes o me tas a cum plir por una bi blio te ca guar dan una es trecha vinculación con el comportamiento de los materiales documen ta les y el uso que se pre ten de ha cer de los mis mos, de ahí que la prioridad o formas que pueda adoptar la estructura técnicade una biblioteca está en función del uso y valoración de los materiales documentales que maneja.

En torno a la biblioteca se presenta un conjunto indeterminado de interacciones universales derivadas de la dinámica cultural, es una en ti dad su je ta a todo tipo de es tímu los y una ma ne ra de entenderla y explicarla es aislando las diversas funciones que la componen. Sin embargo, no se debe perder de vista que cualquier ex pli ca ción acer ca de fun cio nes es pe cífi cas de la bi blio teca únicamente adquiere significado si se encuadra dentro de un mar co de re fe ren cia que per mi ta ubi car el fe nó me no que se tra ta de explicar como parte del todo que conforma la biblioteca.

\section{Los proce dimien tos téc ni cos comofac tor de terminan te en la construcción de modelos bibliotecarios}

La biblioteca aun en sus aspectos estrictamente técnicos se encuen tra li ga da a con tex tos so cia les que la do mi nan, como se ña la Ziman(p.135), la es truc tu ra in te lec tual im plíci ta en el ca tálo goe ín di ces de una bi blio te ca, da sen ti do a la in ves ti ga ción del pa sado, y mo ti vó a la in ves ti ga ción del fu tu ro, al cum plir una fun ción fundamentaldentro del ci clo pro pio de la pu bli ca ción y di fu sión deconocimientos (véase Moles). La acumulación de "literatura primaria" que guardan las bibliotecas constituye un archivo de co no ci mien tos, pero éste se ría inú til sin un buen ca tá lo go o un índice. Es de cir, no po de mos des vin cu lar las fun ciones so cia les de la biblioteca de los conceptos técnicos que ayudan a conformar la organización de sus materiales documentales.

Un ejem plo de cómo de ter mi na dos proce di mien tos téc ni cos encaminados a facilitar la circulación documental, influyen sobre la conceptualización de la biblioteca lo constituye la idea del control bibliográfico. Las limitaciones de espacio físico para el almacenamien to de materiales documentales y la dis ponibilidad fi nan cie ra para su ad qui si ción, au na do a la im po si bi li dad de predecir los requerimientos futuros de información, hicieron poco práctico depender exclusivamente del reducido campo abarcable por una bi blio teca. Fue en ton ces im pres cin dible crear fór mur las que permitieran introducir procesos de almacenamiento y recuperación documental similares para todas las bibliotecas.

Tratar de responder a esta necesidad en el ám bi to de la co mu nicación del conocimiento favoreció el fortalecimiento de la idea del control bibliográfico, cuya intención básica es generar un orden téc ni co que uni for ma se el re gis tro de da tos de la des cripción ca ta lo gráfi ca, con ob je to de crear me ca nis mos para el in tercambio de registros catalográficos y facilitar con ello la preparación y consulta de listas o catálogos elaborados por distintas entidades (Davinson, p.8). Obtener un adecuado control biblio gráfi co con du jo a pro mo ver en elám bi to so cial el con cep to de organizaciónbibliográfica, como un con jun to de con ve nios 
y acuerdos técnicos orientados a uniformar el manejo de datos catalográficos, y promoverlacoo peración en trebibliotecas con base en un orden técnico normativo (Davinson, p.8).

Para cumplir eficientemente las propuestas del control y organización bibliográfica, las técnicas de descripción y organización catalográfica fincaron su principal fundamento en el concepto de la normalización. Las prácticas de normalización generaron toda una serie de reglas y normas, como medio para proveer la necesaria "uniformidad y eficiencia" en el intercambio de datos y la interconexión de diversos sis te mas de al macenamiento y recuperación de información catalográfica.

Con la in cor po ra ción de la idea de nor ma li za ción las téc ni cas de ca ta lo ga ción des crip ti va y es que mas de cla sifi ca ción crea ron un peculiar, eficien te e in fle xible conjun to de proce dimien tos téc ni cos, que subordinan cualquier objetivo al exacto cumplimiento de la nor ma para ob te ner la tan anhe la da uni for mi dad ca ta lo gráfica.

El con cep to de con trol bi blio grá fi co al tra tar de abar car un ám bito universal,promovió que los ideales técnicos de organización y manejo de materiales documentales trascendieran las reducidas ex pec ta tivas de una or ga ni zación biblio te cariain de pen diente y fomentara el incremento de relaciones entre bibliotecas. Surgieron entonces nuevos conceptos, tales como sistema bibliotecario, catálogos colectivos, redes de diseminación de información, etc., con la pretensión de alcanzar un control bibliográfico encaminado a organizar el registro de materiales do cu men ta les de todo tipo, dis po ni bles a tra vés de un am plio número de bibliotecas.

El planteamiento de un control bibliográfico universal, se susten ta en una ló gi ca de or ga ni za ción téc ni ca que su bor di na los requerimientosparticulares de una biblio te ca a las ne cesida des de la creación de una estructura de registro y di-seminación de información catalográfica común a todas las bibliotecas.

El propósito de utilizar estructuras de organización técnica comu nes a to das las bi blio te cas se apo yó fir me men te en idea les sociales que asignan a la biblioteca un papel fundamental para ob te ner la equi dad y li ber tad en el ac ce so al co noci mien to (véa se ALA).

Aun que en la prác ti ca ha crea do de se qui li brios res pec to a la disponibilidad y circulación de materiales documentales, originados por la pre sen cia de cen tros ca pa ces de con ver tir se en nú cleos rec tores del con trolbiblio gráfi co da dos sus re cur sos téc ni cos y/o financieros.

Las es truc tu ras del con trol bi blio grá fi co, se im po nen comoideales sociales de organización bibliotecaria, y están orientadas al cumplimiento de una doble finalidad, a saber:

crear las condiciones técnicas indispensables para que la organización de una biblioteca responda a sus necesidades y propósitos, y
- formalizar mecanismos para que la organización interna de una bi blio te ca res pon da a las me tas pre es ta ble ci das por la plani fi ca ción bi blio te ca ria de ri va da de la idea del con trol bi bliográfico.

Las estructuras creadas por el con trol y la or ga ni za ción bi-bliográfica, no sa tis fa cen to das las ne ce si da des en cuan to a localización de información, porque como cuestiona Escarpit (p.198), "de he cho el ren di mien to de este tra ba jo es ex tre ma da men te débil y el esfuerzo que cuesta al catalogador no está compensado por la ayuda que aporta al lector. Este último sólo encuentra lo quebuscaen elcatálogocuandolohaiden tificadopreviamentey esto es lo más difícil."

Un sistema de control bibliográfico nos sirve para lograr que la so cie dad pue da pre servar, ab sor ber y usar la in for ma ción que gene ra y aquel la que ha he re da do, así como la que re ci be del ex terior; por tanto no debe de estar exento de juicios de valor, que indiquen lo adecuado o improcedente del sistema.

Estos juicios de valor no deben recaer exclusivamente en cuestiones ais la das de na tu ra le za téc nica, por que se li mi ta ría el análisis al juz gar la ca li dad de los proce di mien tos téc ni cos uti li za dos. Una de las finalidades de las síntesis del conocimientobibliotecoló gi cora di caría en trans for mar la in for ma ción en un pa trón de pensamien tointeligible, que permitavisualizarlasinteracciones de un fe nó me no bi blio te ca rio, para po der de du cirsus ca rac te rísti cas téc ni cas par ti cu la res y sus cuali da des parael cum pli mien to de metas sociales predefinidas.

\section{Del por qué y para qué de la síntesis}

Como los fe nó me nos biblio te carios se com po nen tan to de as pectos estrictamente técnicos, como de finalidades derivadas del con tex to so cial don de se ubi ca la bi blio te ca, es ne ce sa rio ar ti cular el conocimiento bibliotecológico en un cuerpo coherente de ca te go rías a par tir de las cua les sea fac ti ble su bor di nar la téc ni ca a la sa tis fac ción de las necesi da des hu ma nas vi ta les en cuan to al acceso al conocimiento por medio de la biblioteca.

La elaboración de síntesis críticas ayudaría a construir panoramas so bre el proce so de evo lu ción e in te rac cio nes de los fe nó menos bibliotecarios, que servirían como punto de partida para ordenar la investigación en torno a problemas cuya coherencia lógicaenfatice las in te rre la cio nes de los ob je tos de es tu dio de la biblio te co logía, y haga po sible in te grar los re sul ta dos, den tro de un marco coherente de conocimientos bibliotecológicos.

En biblio te co lo gía no se ne ce si ta más y más mis ce lá nea in for mativasin correlacionar, esim pres cin dible ar ti cularel co nocimiento que exis te so bre los fe nó me nos bi blio te ca rios para, al me nos, pro mo ver la pre ci sión de fron te ras cla ras e in te gra ción je rár quica de los objetos de estudio de la bibliotecología.

Con ello se alen ta ría la or ga ni za ción sis te má ti ca de la in ves ti gación, que representaría un potencial para su direccíon, dado que la ce rra du ra ló gi ca a par tir de un con jun to cen tral de axio mas sobre los fe nóme nos biblio te carios, per mi ti ría el or de na mien to de las prioridades y estrategias de investigación, porque una vez 
que se cuenta con una estructura básica, se trataría de llenar los huecos, o de encontrar soluciones a los problemas, y desde un punto de vista estrictamentecientífico no existe mucha diferencia en cuanto a cuál hueco se llena primero.

Lain te gra ción je rár qui ca de los ob je tos de es tu dio de la bi blio tecología per mi tiría una práctica co ti dia na de in ves ti ga ción, sen si ble a la ur gen te ne ce si dad de al can zar un ade cua do ba lan ce en tre el conocimiento bibliotecológico y el meramente pragmático.

Es ne ce sa rio como in di ca Ni gel Ford (p.44), rea li zar un in ten so y sus tan cial diálogo ein te rac ción en tre in ves ti ga ción y práctica. Para ello se pue de partir de sín te sis críticas del co no ci mien to biblio te coló gi co, que proporcionen los marcos dereferenciaútiles para ubicar y orientar la investigación técnica de problemas es-

\section{REFERENCIAS BIBLIOGRAFICAS}

(ALA)AMERICANLIBRARY ASSOCIATION. Co mis sion on Free dom and Equa lity of Ac cess to In for mation. Free dom and equality of access to information. Chicago: ALA, 1986. 124 p.

CASEY, Ma rion. Effi ciency, Taylorism, and Libra ries in Progres si ve Ame ri ca. EN Libraries \& Cultura. Edi ted by Do nald G. Davies, Jr. Austin, Texas: Univ. Of Texas Press, 1981. p. 265- 279, Reprinted from Journal of Library History, v. 16 , n. 1 \& 2.

DA VINSON, Donald. Bibliographic Control. London: Linnet Books, 1975. 124 p.

ESCARPIT, Robert. Teoría general de la información y la comunicación. 2a. ed. Barcelona: Icaria, 1981.318 p.

FORD, Nigel. Re search and prac ti ce in li bra rianship: A cog ni ti ve view EN Current trends in information: Research and theory. Edited by Bill Katz and Robin Kinder. New York: Haworth Press, 1987. p. 21-47.

HEMPEL, Carl G.As pects of scien tific ex pla na tion and other es says in the phi losophy of scien ce. New York: Free Press, 1965

HOUSER, L. Documents: The do main of library and in for ma tion science. LibraryandInformation Science Re search, April-june, 1986, v. 8, n. 2, p. 163-189.

MANSFIELD, Una.The wi der sco pe of in for ma tion re search. EN Current trends in information: Research and theory. Edited by Bill Katz and Robin Kinder. New York: Haworth Press, 1987. p. 49- 94

MILANESI, Luiz. Ordenar para desordenar. Sao Paulo,Brasil: Editora Brasilense, 1986.

MOLES, Abraham A. Sociodinámica de la cultura. Versión castellana de Víctor Aizenman, Buenos Aires: Paidos, 1978. 334 p.

NITECKI, Joseph Z.An idea of Librarianship: An Outline for a Root-Methaphor Theory in Library Science. EN Libraries \& Cultura. Edited by Donald G. Da vies, Jr. Aus tin, Te xas: Univ. of Te xas Press, 1981. p. 106- 120, Reprinted from Journal of Library History, v. 16, n. 1 \& 2.

OLIVE, León.Los valores y la investigación social. Investigación Bibliotecológica. Enero-junio 1987, v. 1, n. 2, p. 15-29. 
10. / Investigación Bibliotecológica

RUIZ, Rodríguez Angel.Relación entre las ciencias de la información. Boletín de la Asociación Andaluza de Bibliotecarios, septiembre 1987, año 3, n. 8, p. 5-15.

Vickery, Brian y Alina Vickery. Information science in theory and practice. London: Butterworths, 1987. 384 p.

ZIMAN, John M.Eı Co no ci mien to pú bli co: Un en sa yo so bre la di men sión so cial de la cien cia. Mé xi co: Fon do de Cul tu ra Eco nómica, 1972. 187 p.

ZI MAN (1969), John M. Information, communication, knowledge Nature, 25 oc tu bre 1969, v. 224. Re pro du ci do en: In tro ducc tion to information science/ compiled and edited by Tefko Saracevic. New York: Bowker, 1970. p. 76-84. 